\title{
Improvement of the image quality of random phase-free holography using an iterative method
}

\author{
Tomoyoshi Shimobaba, ${ }^{a}$, Takashi Kakue ${ }^{\mathrm{a}}$, Yutaka Endo ${ }^{\mathrm{a}}$, Ryuji Hirayama ${ }^{\mathrm{a}}$, \\ Daisuke Hiyama $^{a}$, Satoki Hasegawa ${ }^{a}$, Yuki Nagahama ${ }^{a}$, Marie Sano $^{a}$, \\ Minoru Oikawa ${ }^{\mathrm{a}}$, Takashige Sugie ${ }^{\mathrm{a}}$, Tomoyoshi Ito $^{\mathrm{a}}$ \\ ${ }^{a}$ Chiba University, Graduate School of Engineering, 1-33 Yayoi-cho, Inage-ku, Chiba, \\ Japan, 263-8522
}

\begin{abstract}
Our proposed method of random phase-free holography using virtual convergence light can obtain large reconstructed images exceeding the size of the hologram, without the assistance of random phase. The reconstructed images have low-speckle noise in the amplitude and phase-only holograms (kinoforms); however, in low-resolution holograms, we obtain a degraded image quality compared to the original image. We propose an iterative random phase-free method with virtual convergence light to address this problem.

Keywords: Computer-generated hologram, Electroholography, Hologram, Holography, Holographic projection, Kinoform, Phase-only hologram
\end{abstract}

\section{Introduction}

Digital holographic display is a promising technique, because a wavefront of light scattered from an object can be appropriately reconstructed from the display; therefore, this property will enable the realization of an ideal threedimensional display and projector. Holographic projections [1, 2, 3, 3, 4, 5] have unique properties, including multi-projection [6] ( by which a multi-image is projected on multiple screens), projection on screens of arbitrary surface, and lensless zoom-able holographic projection [7, 8, 9]. The lensless zoomable holographic projection will lead to the development of an ultra-small

\footnotetext{
*Tel: +81 43290 3361; fax: +81 432903361

Email address: shimobaba@faculty.chiba-u.jp (Tomoyoshi Shimobaba)
} 
projector. Reconstructed images exceeding the hologram size, in general, require the random phase; however, this causes considerable problems of speckle noise.

There are well-known methods for improving the random phase appliedholograms: the Gerchberg-Saxton (GS) algorithm [10, 11], the multi-random phase method [12], the one-step-phase-retrieval method (OSPR) [13], and

pixel separation methods [14, 15, 16]. The multi-random phase and pixel separation methods require display devices with high-speed refresh rates. Conversely, random phase-free methods have also been proposed, for example, the error diffusion [13, 17, 18, 19, 20] and down-sampling methods [21]. These methods can reconstruct clear images; however, the size of the reconstructed image cannot exceed the size of the hologram, because light fron the object does not spread widely [5]. Therefore, these methods cannot be used for lensless zoom-able holographic projections.

Recently, new random phase-free methods using virtual special convergence light for amplitude and phase-only holograms (kinoforms) have been proposed [22, 23, 24]. Without the assistance of the random phase, this method can reconstruct images that exceed the hologram's size with lowspeckle noise; however, in low-resolution holograms, we obtain a degraded image quality by ringing artifacts, as will be shown in the next section.

In this paper, we propose an iterative random phase-free method with virtual convergence light to address this problem. Sections 2 and 3 describe the proposed method and the results of simulation performed using it. Section 4 concludes this work.

\section{Proposed method}

The random phase-free method using virtual convergence light is outlined in [22, 23, 24]. The calculation setup is shown in Fig. 1. This method is applicable to amplitude computer-generated holograms (CGHs) and kinoforms. Instead of using the random phase, the complex amplitude on the image plane $u_{i}\left(x_{i}, y_{i}\right)$ is multiplied using virtual convergence light given by

$$
w\left(x_{i}, y_{i}\right)=\exp \left(-i \pi\left(x_{i}^{2}+y_{i}^{2}\right) / \lambda f_{i}\right),
$$

where $f_{i}=z_{1}+z_{2}$ is the focal length, $z_{1}$ is the distance between the focus point of the convergence light and the hologram, and is set to the distance at which the hologram just fits to the cone of the convergence light, and $z_{2}$ is the distance between the object and hologram. 


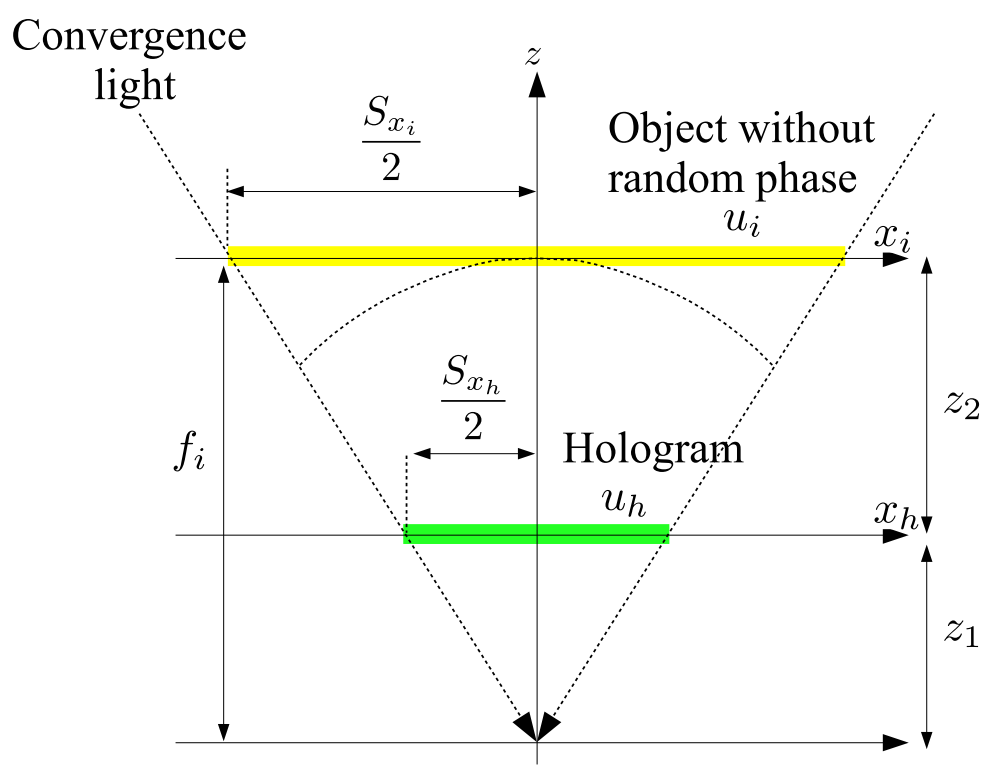

Figure 1: Calculation setup for the random phase-free method using virtual convergence light.

Here we describe how to determine $f_{i}$. Using a simple geometric relation, we can derive $S_{h} / 2: S_{i} / 2=z_{1}: f_{i}$ where the areas of the image and the amplitude CGH (or kinoform) are given by $S_{i} \times S_{i}$ and $S_{h} \times S_{h}$, respectively. Therefore, we obtain:

$$
f_{i}=z_{2} /\left(1-S_{h} / S_{i}\right) .
$$

To avoid overlap between the reconstructed image and the 0-th order light, the original object must be shifted from the optical axis by a distance of $o$. Owing to the addition of this shift amount, the focal length of the convergence light is expressed as

$$
f_{i}=z_{2} /\left(1-S_{h} /\left(S_{i}+2 o\right)\right) .
$$

We calculate the complex amplitude in the hologram plane by

$$
u_{h}\left(x_{h}, y_{h}\right)=\operatorname{Prop}_{z_{2}}\left\{u_{i}\left(x_{i}, y_{i}\right) w\left(x_{i}, y_{i}\right)\right\},
$$

where $\operatorname{Prop}_{z_{2}}\{\cdot\}$ denotes the diffraction calculated at the propagation distance, $z_{2}$. The following equation is used to calculate the amplitude CGH, $I\left(x_{h}, y_{h}\right)$ from $u_{h}\left(x_{h}, y_{h}\right)$ :

$$
I\left(x_{h}, y_{h}\right)=\Re\left\{u_{h}\left(x_{h}, y_{h}\right)\right\},
$$


where $\Re\{\cdot\}$ denotes the real part of $u_{h}\left(x_{h}, y_{h}\right)$. In calculating the kinoform, $\theta\left(x_{h}, y_{h}\right)$, from $u_{h}\left(x_{h}, y_{h}\right)$, the following equation is used:

$$
\theta\left(x_{h}, y_{h}\right)=\arg \left\{u_{h}\left(x_{h}, y_{h}\right)\right\}
$$

where $\arg \{\cdot\}$ indicate the use of only the argument of the complex amplitude. Unfortunately, in kinoforms, the reconstructed images using the random phase-free method are heavily degraded. This is a common problem of kinoforms [19, 20, 21]. To overcome this problem, we apply the error diffusion method [19, 20] to the random phase-free kinoform as follows:

$$
\theta\left(x_{h}, y_{h}\right)=\operatorname{ED}\left\{\theta\left(x_{h}, y_{h}\right)\right\}
$$

where $\operatorname{ED}\{\cdot\}$ denotes the error diffusion operator. The details of the error diffusion are described in Section 2.1. For the details of the random phase free-amplitude CGH and kinoform, refer Ref. [22, 23].

Figure 2 shows reconstructed images from the amplitude CGH and kinoform. Figures 2(a) and 2(c) are the reconstructions from the amplitude CGHs of the images "Lena" and "Mandrill', respectively. Figures 2(b) and 2(d) are the reconstructions from the kinoforms of the images, respectively. These reconstructed images do not have the speckle noise induced by the random phase; however, ringing artifacts overlap in these reconstructed images.

\subsection{Improved image quality using an iterative method}

To overcome the problem with ringing artifacts problem in the reconstructed images, we apply an iterative enhancement of the random phase-free method. Figures 3 and 4 show the improved method for amplitude CGHs and kinoforms using the iterative algorithm, respectively. The kinoform yielded by the iteration method is almost the same as that in Fig. 3, except for the error diffusion. The calculation steps are as follows:

1. We start the iteration by multiplying the virtual convergence light $w\left(x_{i}, y_{i}\right)$ by the original image $a_{i}\left(x_{i}, y_{i}\right)$. The image plane is denoted by $u_{i}\left(x_{i}, y_{i}\right)=a_{i}\left(x_{i}, y_{i}\right) w\left(x_{i}, y_{i}\right)$.

2. We calculate the scaled and shifted diffraction, which can be used to obtain light propagation at different sampling pitches on the image and hologram, as well as the off-axis propagation, by $u_{h}\left(x_{h}, y_{h}\right)=$ $\operatorname{Prop}_{z_{2}}\left\{u_{i}\left(x_{i}, y_{i}\right) w\left(x_{i}, y_{i}\right)\right\}$. The diffraction we used is ARSS Fresnel diffraction [25], which is a scaled and shifted diffraction. The sampling 


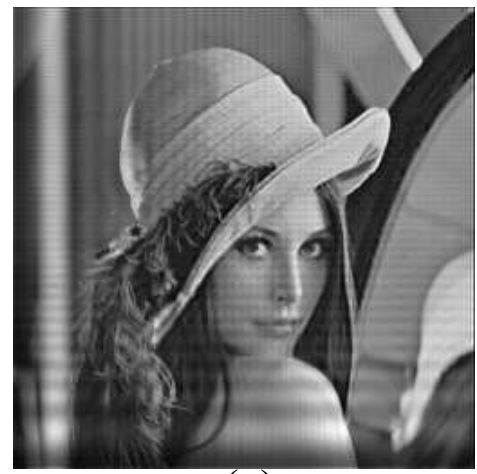

(a)

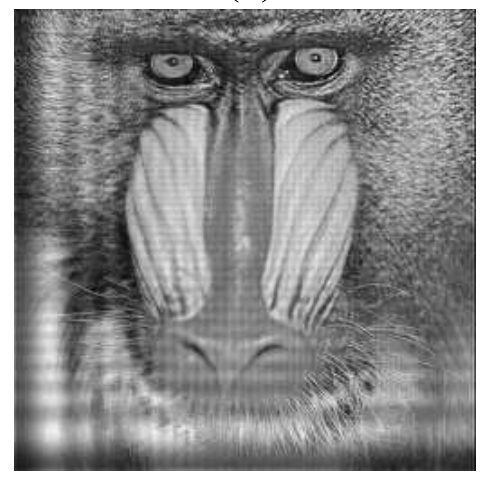

(c)

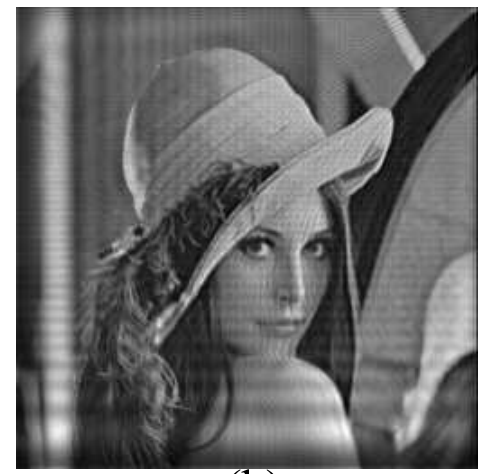

(b)

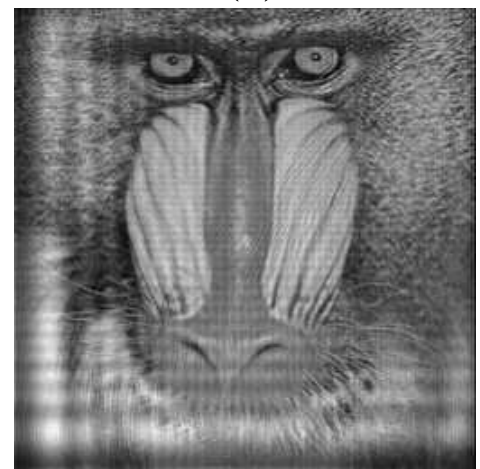

(d)

Figure 2: Reconstructed images from amplitude CGH and kinoform. (a) and (c) reconstructed images from amplitude CGHs: (b) and (d) reconstructed images from kinoform: These reconstructed images are contaminated by ringing artifacts.

pitches on the image and hologram planes are set to $p_{i}$ and $p_{h}$, respectively. The amount of shift away from the optical axis on the image plane is $\left(o_{x}, o_{y}\right)$.]

3. We extract only the real part of $u_{h}\left(x_{h}, y_{h}\right)$ for the amplitude CGH or only the argument of $u_{h}\left(x_{h}, y_{h}\right)$ for the kinoform (which represents constraints in the hologram plane).

$$
g\left(x_{h}, y_{h}\right)=\left\{\begin{array}{lll}
\Re\left\{u_{h}\left(x_{h}, y_{h}\right)\right\} & (\text { For amplitude CGH) (8a) } \\
\operatorname{ED}\left\{\arg \left\{u_{h}\left(x_{h}, y_{h}\right)\right\}\right\} & (\text { For kinoform) } & (8 \mathrm{~b})
\end{array}\right.
$$

4. We back-propagate $u_{h}\left(x_{h}, y_{h}\right)$ to the image plane, $u_{i}^{\prime}\left(x_{i}, y_{i}\right)=\operatorname{Prop}_{-z_{2}}\left\{g\left(x_{h}, y_{h}\right)\right\}$ by ARSS Fresnel diffraction. If we use the offset parameter, the shift amount away from the optical axis should be set to $\left(-o_{x},-o_{y}\right)$. 


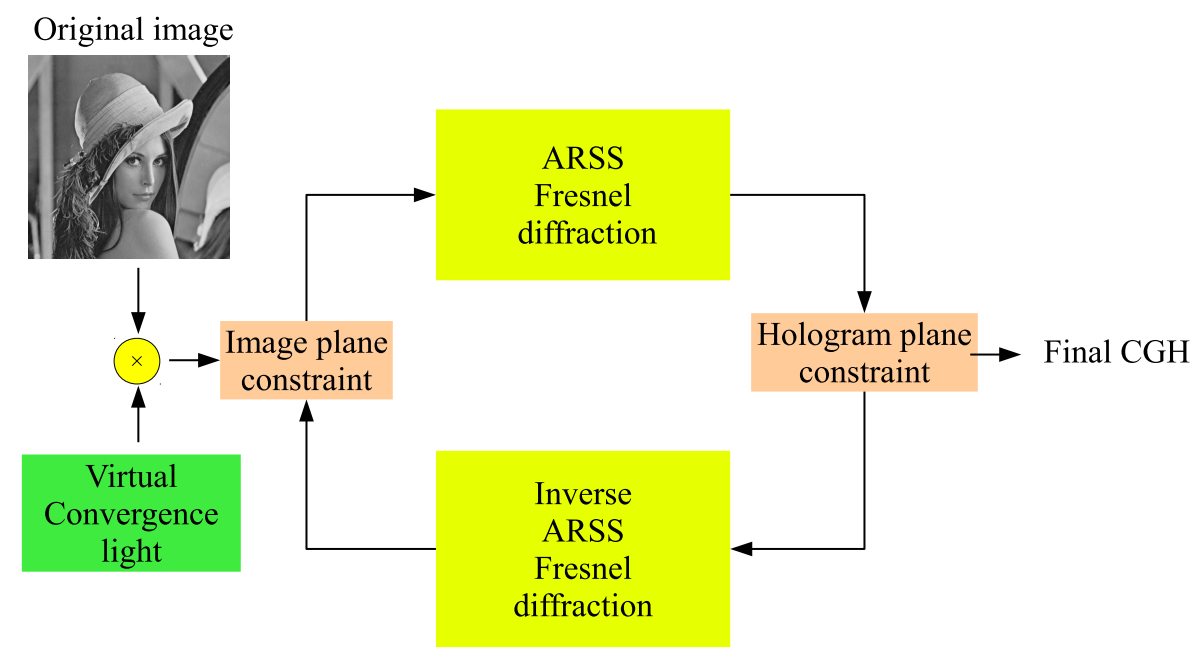

Figure 3: Iterative method for amplitude CGH.

5. We update only the amplitude of $u_{i}^{\prime}\left(x_{i}, y_{i}\right)$ according to

$$
u_{i}\left(x_{i}, y_{i}\right)=a_{i}\left(x_{i}, y_{i}\right) u_{i}^{\prime}\left(x_{i}, y_{i}\right) /\left|u_{i}^{\prime}\left(x_{i}, y_{i}\right)\right|
$$

This is the constraint in the image plane. Subsequently, we obtain the new image plane $u_{i}\left(x_{i}, y_{i}\right)$.

We repeat the above iteration steps (2) to (5) until reaching the preset iteration number or a good image quality.

In the step 3, the Floyd-Steinberg error diffusion method we used was calculated using

$$
\begin{aligned}
\theta\left(x_{h}, y_{h}+1\right) & \leftarrow \theta\left(x_{h}, y_{h}+1\right)+w_{1} e\left(x_{h}, y_{h}\right), \\
\theta\left(x_{h}+1, y_{h}-1\right) & \leftarrow \theta\left(x_{h}+1, y_{h}-1\right)+w_{2} e\left(x_{h}, y_{h}\right), \\
\theta\left(x_{h}+1, y_{h}\right) & \leftarrow \theta\left(x_{h}+1, y_{h}\right)+w_{3} e\left(x_{h}, y_{h}\right), \\
\theta\left(x_{h}+1, y_{h}+1\right) & \leftarrow \theta\left(x_{h}+1, y_{h}+1\right)+w_{4} e\left(x_{h}, y_{h}\right),
\end{aligned}
$$

where the coefficients are $w_{1}=7 / 16, w_{2}=3 / 16, w_{3}=5 / 16$, and $w_{4}=1 / 16$ [19]: $\leftarrow$ indicates that the current value is overwritten, and $e\left(x_{h}, y_{h}\right)=u_{h}\left(x_{h}, y_{h}\right)-$ $\theta\left(x_{h}, y_{h}\right)$. Before applying the error diffusion, we may need to normalize the complex amplitude $u_{h}\left(x_{h}, y_{h}\right)$ by $u_{h}\left(x_{h}, y_{h}\right) \leftarrow u_{h}\left(x_{h}, y_{h}\right) / d$, where $d=$ $\max \left|u_{h}\left(x_{h}, y_{h}\right)\right|$ is the maximum absolute value in the kinoform. 


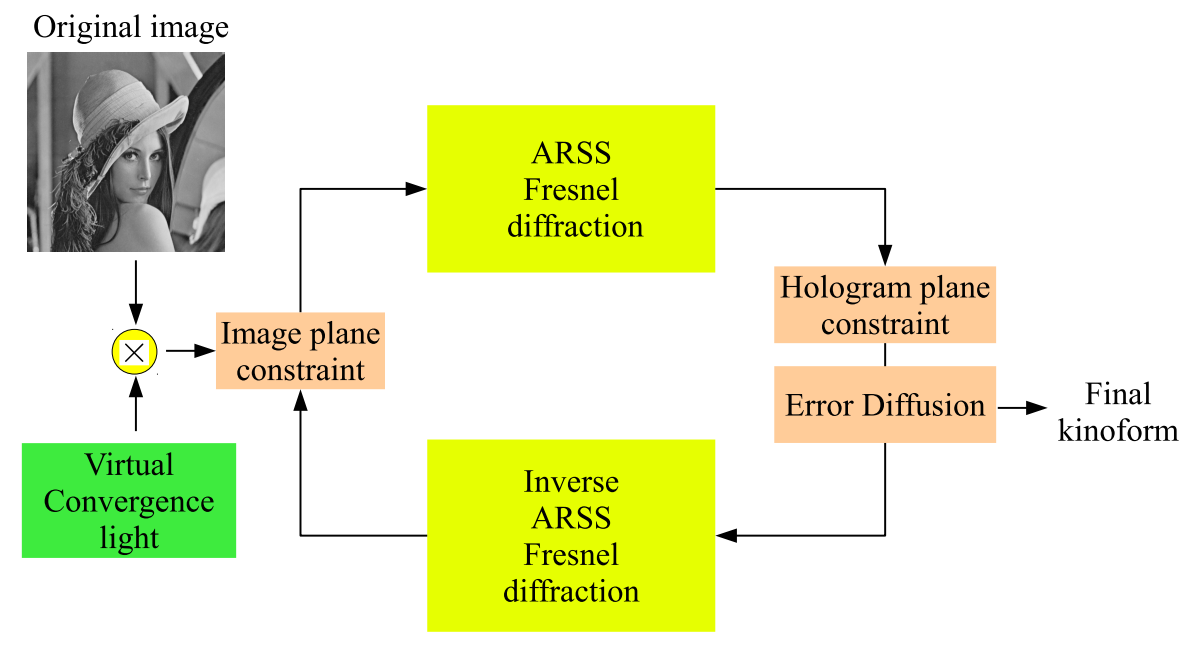

Figure 4: Iterative method for kinoform.

\section{Results}

We show the effectiveness of the proposed method through simulation. The calculation is performed under conditions where the resolution of the images is $2,048 \times 2,048$ pixels, the wavelength is $532 \mathrm{~nm}$, the pixel pitches on the image plane and the hologram plane are $p_{i}=8 \mu \mathrm{m}$ and $p_{h}=4 \mu \mathrm{m}$, respectively, the distance between the image and hologram planes is $1 \mathrm{~m}$, and the amount of shift away from the optical axis is $\left(o_{x}, o_{y}\right)=(20 \mathrm{~mm}, 20 \mathrm{~mm})$.

The upper images in Fig. [5 show reconstructions of the image "Lena" from amplitude CGHs, and the lower images show reconstructions kinoforms, which are generated by the proposed method. Increasing the iteration number results in improvement of the image quality in both amplitude CGH and kinoform. Figure [ 6 shows the peak signal-to-noise ratio (PNSR) between the original image and the reconstructed image when increasing the number of iteration. The blue and green lines indicate the PSNRs for the amplitude CGHs and kinoforms, respectively. The improvement effect converges at five iterations, and the PSNRs of the amplitude CGH and kinoform are about 30 $\mathrm{dB}$ and $27 \mathrm{~dB}$, respectively.

Figure 7 shows the reconstructions of the image "Mandrill". The upper and lower images are obtained from amplitude CGHs and kinoforms generated by the proposed method, respectively. Figure 8 shows the PNSR between the original and the reconstructed images when increasing the number of iteration. The improvement effect is the same as that in Fig, 6 , and the 
PSNRs at five iterations of the amplitude CGH and kinoform are about 23 $\mathrm{dB}$ and $22 \mathrm{~dB}$, respectively. Overall, the proposed method greatly improves the image quality.

\section{Reconstructed images from amplitude CGHs}

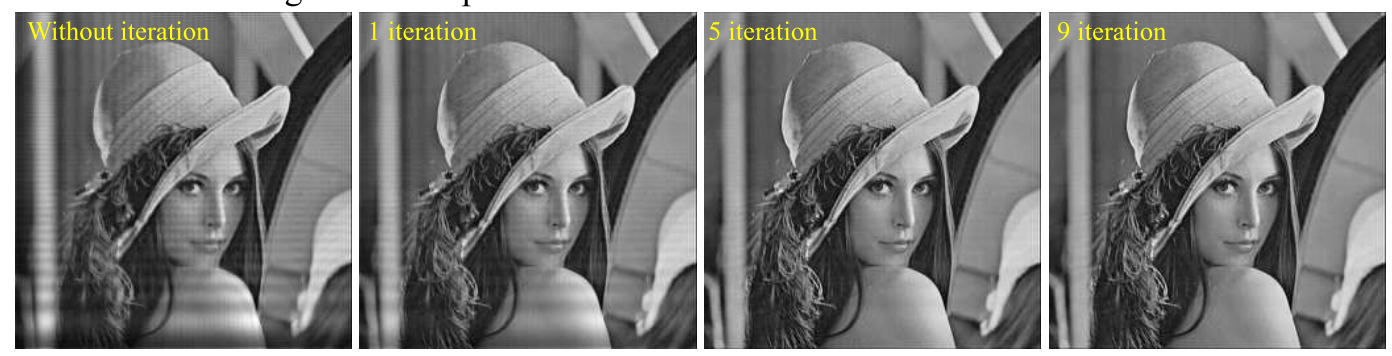

Reconstructed images from amplitude kinoforms
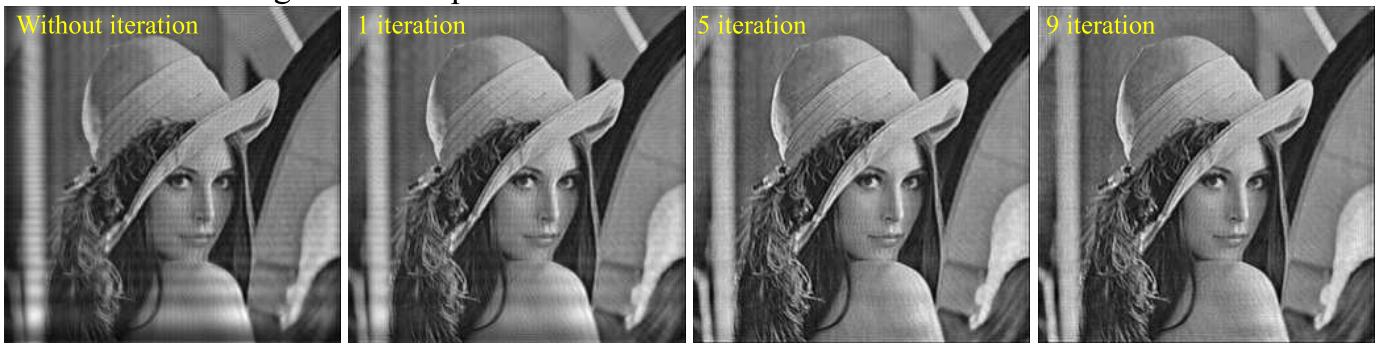

Figure 5: Reconstructions of the image "Lena". The upper and lower images are reconstructed from amplitude CGHs and kinoforms, respectively. They are generated by the proposed method.

Figure 9 shows the reconstructed images from amplitude CGHs with random phase (left) and with the proposed method (right) at five iterations. The PSNRs for the left and right images are $9.8 \mathrm{~dB}$ and $30.5 \mathrm{~dB}$, respectively. Figure 10 shows the reconstructed images from kinoforms with random phase (left), with the proposed method not applying the error diffusion (middle) and with the proposed method using the error diffusion (right) at five iterations. The PSNRs for the left and right images are $9.0 \mathrm{~dB}, 9.4 \mathrm{~dB}$, and 27.1 $\mathrm{dB}$, respectively.

\section{Conclusion}

We proposed an iterative technique for generating amplitude CGHs and kinoforms using the random phase-free method with virtual convergence light. The proposed method succeeded in mitigating ringing artifacts in the reconstructed images. 


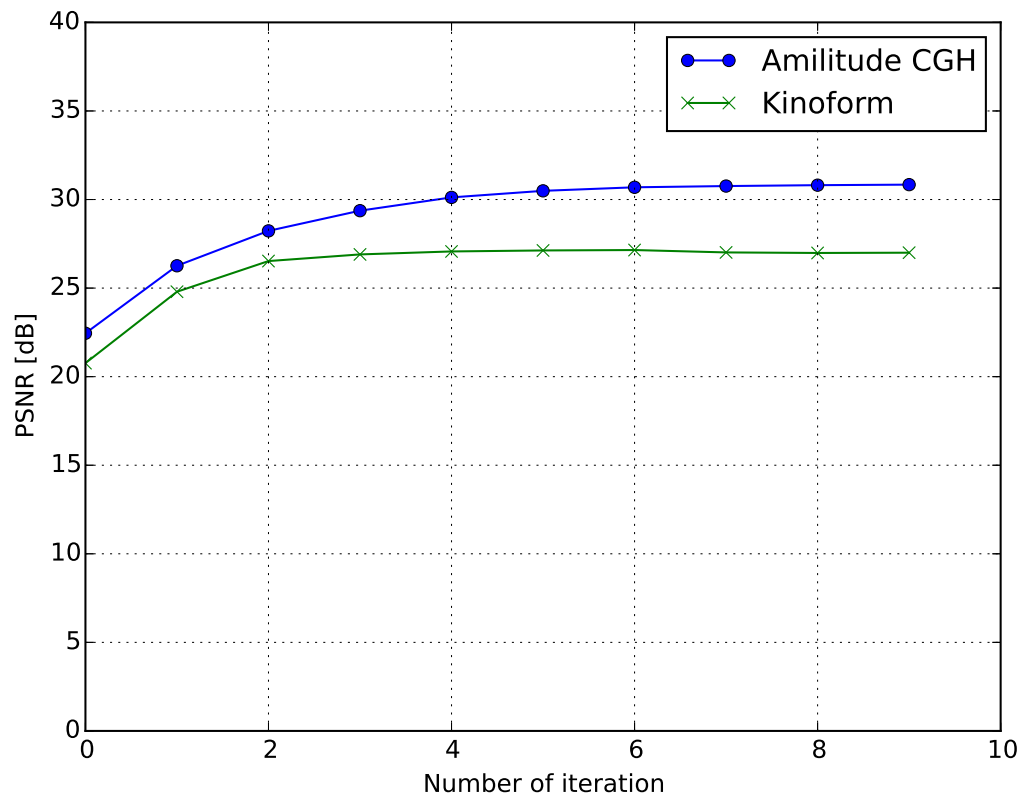

Figure 6: PSNRs for the reconstructed images of "Lena" from the amplitude CGHs and kinoforms.

\section{Acknowlegement}

This work is partially supported by JSPS KAKENHI Grant Numbers 25330125 and 25240015, and the Kayamori Foundation of Information Science Advancement and Yazaki Memorial Foundation for Science and Technology.

\section{References}

[1] E. Buckley, "Holographic Laser Projection," J. Display Technol. 99, 1-6 (2010).

[2] E. Buckley, "Holographic projector using one lens, " Opt. Lett. 35, 3399-3401 (2010).

[3] M. Makowski, M. Sypek, and A. Kolodziejczyk, "Colorful reconstructions from a thin multi-plane phase hologram, " Opt. Express 16, 1161811623 (2008). 
Reconstructed images from amplitude CGHs
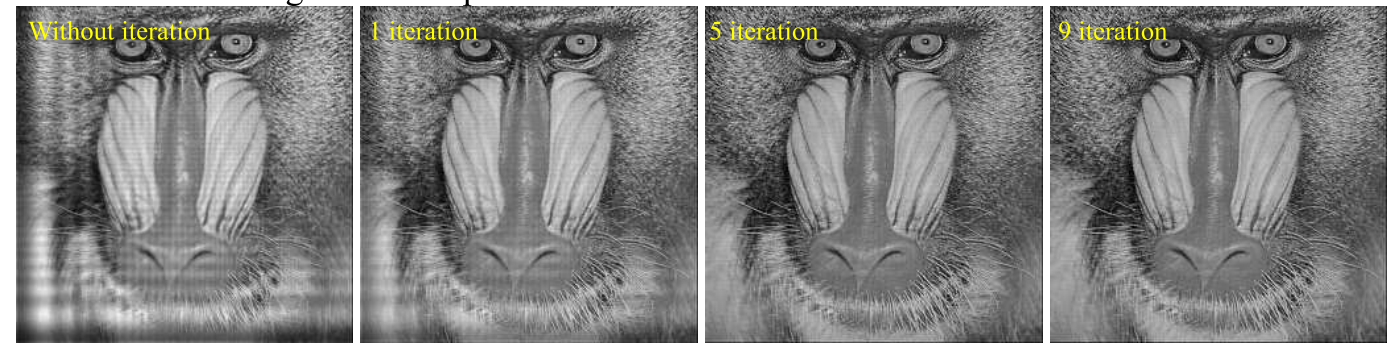

Reconstructed images from amplitude kinoforms
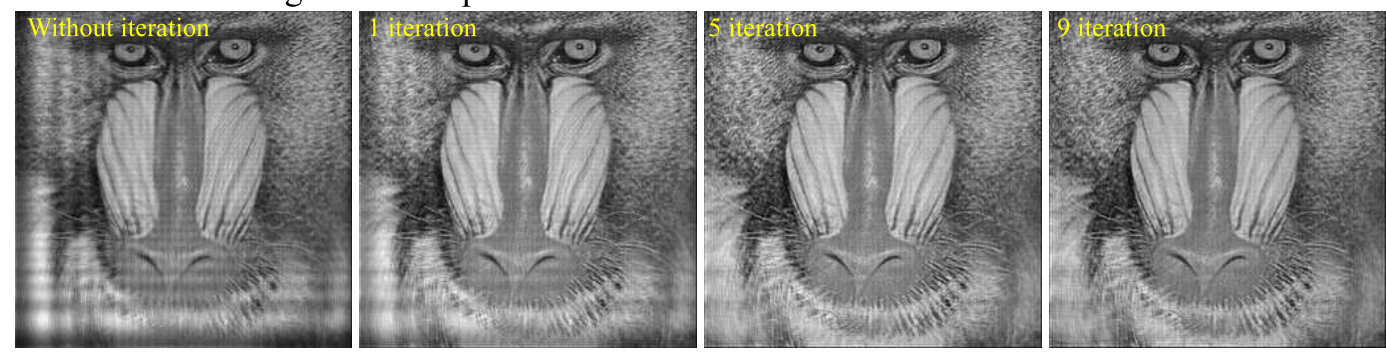

Figure 7: Reconstructions of the image "Mandrill". The upper and lower images are reconstructed from amplitude CGHs and kinoforms, respectively. They are generated by the proposed method.

[4] M. Makowski, M. Sypek, I. Ducin, A. Fajst, A. Siemion, J. Suszek, and A. Kolodziejczyk, "Experimental evaluation of a full-color compact lensless holographic display," Opt. Express 17, 20840-20846 (2009).

[5] T. Shimobaba, T. Kakue, and T. Ito, "Real-time and low speckle holographic projection," IEEE International Conference on Industrial Informatics INDIN' 15 (Special Session on Digital Holography for Industrial Applications) (2015).

[6] T. Shimobaba, M. Makowski, T. Kakue, N. Okada, Y. Endo, R. Hirayama, D. Hiyama, S. Hasegawa, Y. Nagahama, and T. Ito, "Numerical investigation of lensless zoomable holographic projection to multiple tilted planes," Opt. Commun. 333, 274-280 (2014).

[7] T. Shimobaba, T. Kakue, N. Masuda, and T. Ito, "Numerical investigation of zoomable holographic projection without a zoom lens," JSID 20, 533-538 (2012).

[8] T. Shimobaba, M. Makowski, T. Kakue, M Oikawa, N. Okada, Y. Endo, 


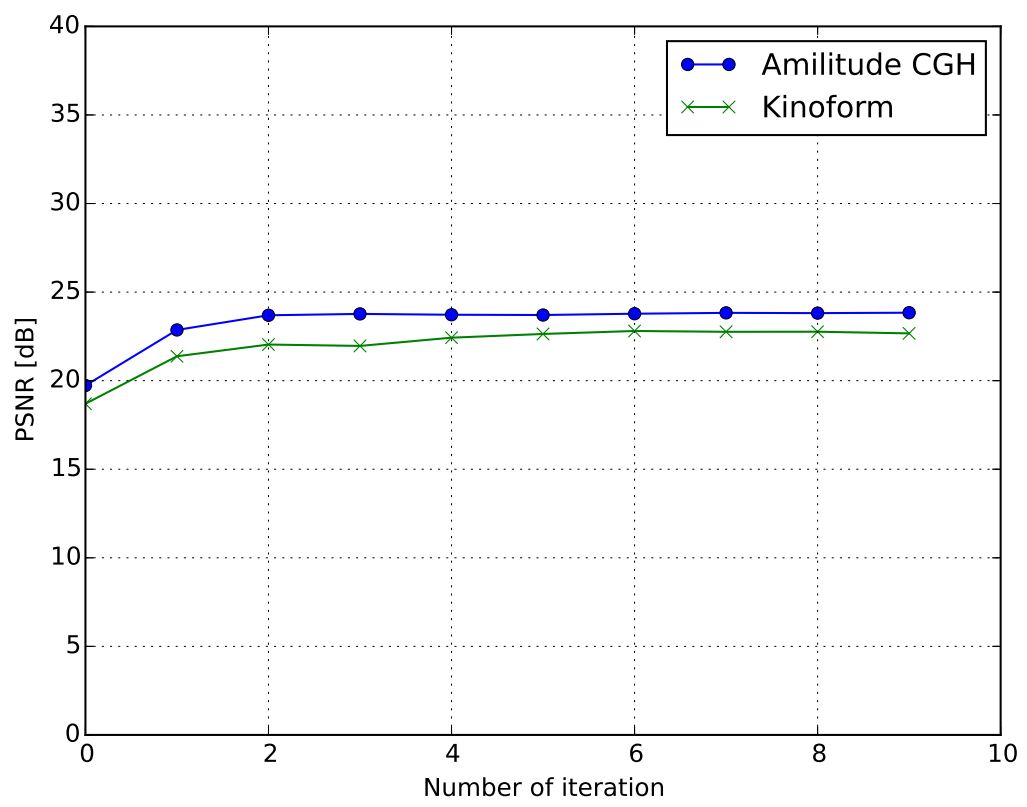

Figure 8: PSNRs for the reconstructed images of "Mandrill" from the amplitude CGHs and kinoforms.

R. Hirayama, and T. Ito, "Lensless zoomable holographic projection using scaled Fresnel diffraction," Opt. Express 21, 25285-25290 (2013)

[9] I. Ducin, T. Shimobaba, M. Makowski, K. Kakarenko, A. Kowalczyk, J. Suszek, M. Bieda, A. Kolodziejczyk, and M. Sypek, "Holographic projection of images with step-less zoom and noise suppression by pixel separation," Opt. Commun. 340, 131-135 (2015).

[10] R. W. Gerchberg and W. O. Saxton, "A Practical Algorithm for the Determination of Phase from Image and Diffraction Plane Pictures," Optik 35, 237-246 (1972).

[11] M. Makowski, M. Sypek, A. Kolodziejczyk, and G. Mikula, "Threeplane phase-only computer hologram generated with iterative Fresnel algorithm," Opt. Eng. 44, 125805 (2005).

[12] J. Amako, H. Miura, and T. Sonehara, "Speckle-noise reduction on 

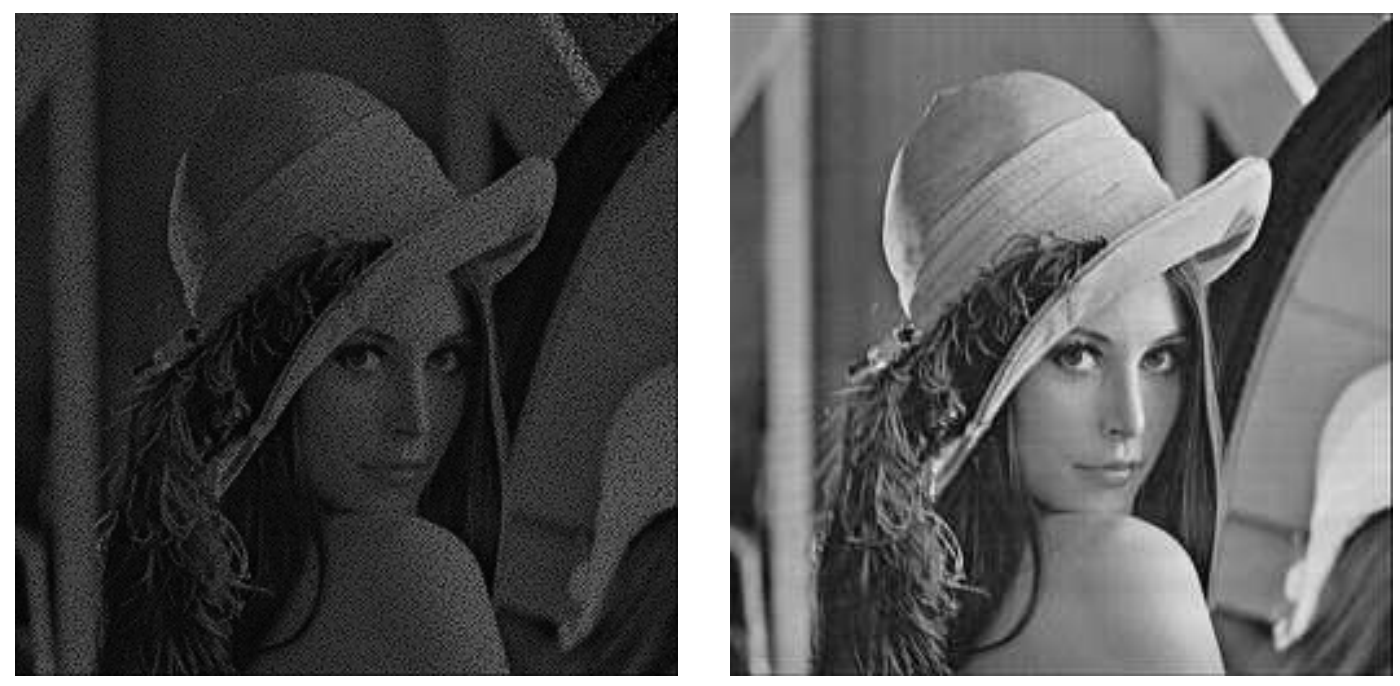

Figure 9: Reconstructed images at five iterations. With random phase (left). With the proposed method (right).

kinoform reconstruction using a phase-only spatial light modulator," Appl. Opt. 34, 3165-3171 (1995).

[13] E. Buckley, "Real-Time Error Diffusion for Signal-to-Noise Ratio Improvement in a Holographic Projection System," J. Display Technol. 7, 70-76 (2011).

[14] Y. Takaki and M. Yokouchi, "Speckle-free and grayscale hologram reconstruction using time-multiplexing technique," Opt. Express 19, 75677579 (2011).

[15] M. Makowski, "Minimized speckle noise in lens-less holographic projection by pixel separation," Opt. Express 21, 29205-29216 (2013).

[16] Y. Mori, T. Fukuoka, and T. Nomura, "Speckle reduction in holographic projection by random pixel separation with time multiplexing," Appl. Opt. 53, 8182-8188 (2014).

[17] R. Eschbach, "Comparison of error diffusion methods for computergenerated holograms," Appl. Opt. 30, 3702-3710 (1991).

[18] S. Weissbach, Frank Wyrowski, and Olof Bryngdahl, "Error-diffusion 

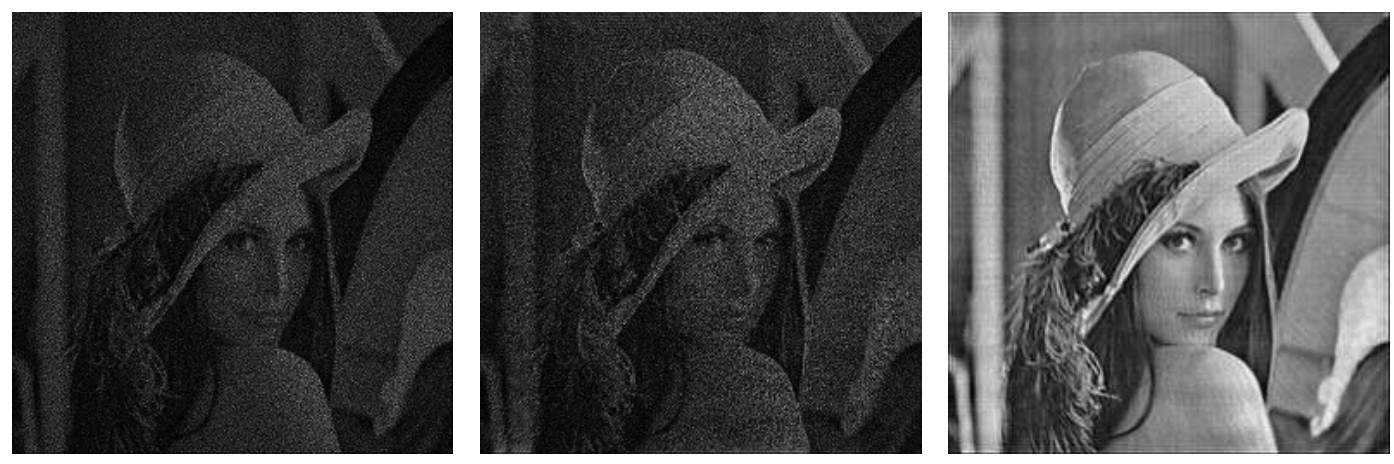

Figure 10: Reconstructed images at five iterations. With random phase (left). With the proposed method not applying the error diffusion (middle). With the proposed method using the error diffusion (right).

algorithm in phase synthesis and retrieval techniques," Opt. Lett. 17, 235-237 (1992)

[19] P. W. M. Tsang and T. -C. Poon, "Novel method for converting digital Fresnel hologram to phase-only hologram based on bidirectional error diffusion," Opt. Express 21, 23680-23686 (2013)

[20] P. W. M. Tsang, A. S. M. Jiao, and T.-C. Poon, "Fast conversion of digital Fresnel hologram to phase-only hologram based on localized error diffusion and redistribution," Opt. Express 22, 5060-5066 (2014)

[21] P. W. M. Tsang, Y. -T. Chow, and T. -C. Poon, "Generation of phaseonly Fresnel hologram based on down-sampling," Opt. Express 22, 25208-25214 (2014).

[22] T. Shimobaba and T. Ito, "Random phase-free computer-generated hologram," Opt. Express (accepted).

[23] T. Shimobaba, T. Kakue, Y. Endo, R. Hirayama, D. Hiyama, S. Hasegawa, Y. Nagahama, M. Sano, M. Oikawa, T. Sugie, and T. Ito, "Random phase-free kinoform for large objects," (submitted).

[24] T. Shimobaba, T. Kakue, Y. Endo, R. Hirayama, D. Hiyama, S. Hasegawa, Y. Nagahama, M. Sano, M. Oikawa, T. Sugie, and T. Ito, "Optical encryption for large-sized images using random phase-free methods," (submitted). 
[25] T. Shimobaba, T. Kakue, N. Okada, M. Oikawa, Y. Yamaguchi, and T. Ito, "Aliasing-reduced Fresnel diffraction with scale and shift operations," J. Opt. 15, 075302 (2013). 